\title{
In vitro effect of iron chelators on the growth of Escherichia coli, Staphylococcus epidermidis, Staphylococcus aureus, Yersinia enterocolitica, and Pseudomonas aeruginosa Strains
}

\author{
Kiavash Fekri $^{(\mathbb{D}}$, Abolfazl Khoshde ${ }^{2 *}{ }^{(\mathbb{D}}$, Maryam Rasoulynezhad ${ }^{3}$, Soleiman Kheiri ${ }^{4}$, Afsaneh Malekpour ${ }^{5}$, Behnam Zamanzad ${ }^{6}$ \\ ${ }^{1}$ Assistant Professor, Department of Pediatrics, Shahrekord University of Medical Sciences, Shahrekord, Iran \\ ${ }^{2}$ Professor, Clinical Biochemistry Research Center, Shahrekord University of Medical Sciences, Shahrekord, Iran \\ ${ }^{3} \mathrm{MD}$, Shahrekord University of Medical Sciences, Shahrekord, Iran \\ ${ }^{4}$ Associate Professor, Clinical Biochemistry Research Center, Shahrekord University of Medical Sciences, Shahrekord, Iran \\ ${ }^{5}$ Assistant Professor, Department of Community Medicine, Shahrekord University of Medical Sciences, Shahrekord, Iran \\ ${ }^{6}$ Assistant Professor, Department of Microbiology and Immunology, Shahrekord University of Medical Sciences, Shahrekord, \\ Iran
}

*Corresponding Author: Abolfazl Khoshdel Department of Pediatrics, Shahrekord University of Medical Sciences, Shahrekord, Iran Tel./Fax: +983833338891 Email: nikakhosh@gmail.com

\begin{abstract}
Background and aims:Thalassemia patients are predisposed to septicemia due to iron overload. The iron chelators have a potential role in the treatment of septicemia. This study was conducted to investigate the in vitro effect of iron chelators, deferoxamine (DFO), deferiprone (DFP), and deferasirox (DFS) on the growth of effective bacteria (Staphylococcus aureus, Yersinia enterocolitica, and Pseudomonas aeruginosa) and their synergistic effect with each other and antibiotics.

Materials and Methods: Five bacterial strains were incubated in the presence of seven chelators in Mueller Hinton agar and the number of colonies was recorded for each sample. Antibiotic discs were placed in the plates. Susceptibility to antibiotics was assessed by disc diffusion method (Kirby-Bauer test) and also the zone inhibition around the disc was measured in $\mathrm{mm}$ after incubating the relevant plate at $37^{\circ} \mathrm{C}$ for a day.

Results: The growth of all the studied bacteria increased in the presence of iron and was inhibited in the presence of different chelators. Chelators and antibiotics had synergistic effects on growth inhibition of Escherichia coli, S. aureus, and P. aeruginosa in culture medium, while the growth inhibition of $Y$. enterocolitica was seen in the presence of deferiprone and deferasirox combination.

Conclusion: Chelators in combination with antibiotics exert synergistic effects on growth inhibition of prevalent bacteria (except for $Y$. enterocolitica).

Keywords: E. coli, S. epidermidis, S. aureus, Y. enterocolitica, P. aeruginosa, Thalassemia, Iron chelators
\end{abstract}

Received: 20 April 2019, Accepted: 28 July 2019, ePublished: 30 October 2019

\section{Introduction}

Thalassemia is a genetic blood disorder which is characterized by abnormality in the synthesis of hemoglobin beta chain and ranges from severe to asymptomatic anemia (1). The prevalence of thalassemia varies worldwide. In Iran, 8000 pregnancies per year are estimated to be at risk of having babies with thalassemia (2). Major Thalassemia is considered as a severe form of thalassemia which is manifested within the first two years of life and the affected patients should regularly undergo red blood cell transfusion (1). Blood transfusion in these patients leads to complications of iron overload with high morbidity and mortality if left untreated $(3,4)$.

Iron overload in thalassemia patients could cause bone disorders, endocrine gland disorders (delayed growth, sexual maturation disorders, diabetes mellitus, and failure of thyroid, parathyroid, and adrenal glands), heart problems, and cirrhosis $(1,5,6)$. One of the conditions that could cause death of thalassemia patients is infectious diseases and subsequent septicemia (7-9). In these patients, increase in blood ferritin, repeated venous catheterizations, and splenectomy are considered as major risk factors for septicemia. In addition, these patients are predisposed to infection with gram-positive and -negative bacteria (10, 11).

Although thalassemia declines the quality of life among the affected patients (12), the treatment with different iron chelators enhances life expectancy, decreases iron

(C) 2019 The Author(s); Published by Shahrekord University of Medical Sciences. This is an open-access article distributed under the terms of the Creative Commons Attribution License (http://creativecommons.org/licenses/by/4.0), which permits unrestricted use, distribution, and reproduction in any medium, provided the original work is properly cited. 
overload-associated complications, and promotes health in them $(13,4)$. Some studies have indicated that iron chelators have a potential role in the treatment of septicemia (15). Although iron overload in thalassemia patients is an independent predictive factor for infection, some investigations have demonstrated that bacterial response to iron overload in the body could vary. Accordingly, iron overload causes an increase in the growth of some bacteria and a decrease in the growth of some others. In addition, the type of chelators could contribute greatly to bacterial growth $(16,17)$. It is necessary to investigate new classes of iron chelators recently developed for the treatment of various diseases including thalassemia. It is because their antibacterial activities may be greater than their chelationrelated ones (18).

The findings on the potential viability of iron chelators as therapeutic agents against various bacteria have been inconsistent. Moreover, as bacterial resistance against antibiotics has increased and the synergistic effect of iron chelators and antibiotics has not yet been investigated, this study is conducted to investigate the in vitro effect of iron chelators, deferoxamine (DFO) (Desferal), deferiprone (DFP), and deferasirox (DFS) on the growth of the effective bacteria and their synergistic effect alongside each other and antibiotics in thalassemia patients with septicemia (Escherichia coli, Staphylococcus epidermidis, Staphylococcus aureus, Yersinia enterocolitica, and Pseudomonas aeruginosa).

\section{Materials and Methods}

In this experimental laboratory study, five bacterial samples including E. coli ATCC: 27922, S. aureus ATCC: 27923, S. epidermidis ATCC 12228, Y. enterocolitica ATCC 33669, and P. aeruginosa ATCC: 27853were obtained from Scientific and Industrial Research Co, Tehran, Iran. Then, the samples were cultured on iron-containing and non-iron-

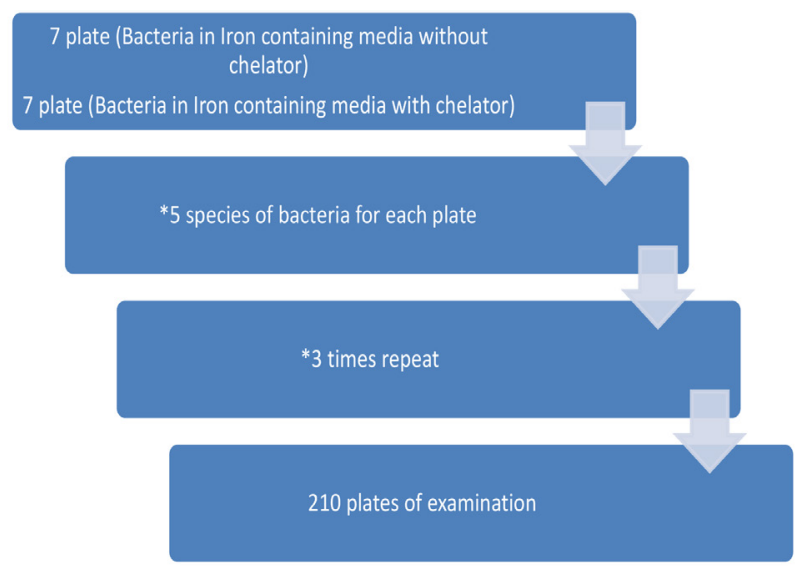

Figure 1. Flowchart of Study Design containing media using standard bacteriologic methods (19). Iron-containing media were divided into two groups. Then, two sets of chelators were individually added as follows: Group 1 without chelators and group 2 containing 3 plates with only one chelator including DFO, DFS, and DFP (Iran Daru Co), as well as 4 plates with a combination of Desferal + DFS, Desferal + DFP, DFP+DFS and Desferal + DFP + DFS. Then, the sensitivity of cultured bacteria to some common antibiotics in iron-containing media with and without chelators was consequently detected (Figures $1,2)$. The details of our procedures were as follows: For the samples with iron, $0.025 \mathrm{~g}$ ferric iron chloride (equivalent to $150 \mu \mathrm{M}$ ferric chloride) was mixed with Mueller-Hinton agar (Merck), left at $121^{\circ} \mathrm{C}$, and autoclaved for 15 minutes. After the temperature declined to $50^{\circ} \mathrm{C}$, the chelators of interest at $100 \mu \mathrm{M} / \mathrm{L}$ concentration were introduced into the medium of interest per molecular mass $(\mathrm{g})$ of each chelator $(20,21)$. Therefore, $0.56 \mathrm{~g} / \mathrm{L}$ DFO, $0.37 \mathrm{~g} / \mathrm{L} \mathrm{DFS}$, and $0.14 \mathrm{~g} / \mathrm{L}$ DFP were added to the culture media.

Bacterial samples in the tubes containing saline and 0.5 McFarland standard media were separated using $10 \mu \mathrm{L}$ sampler while being adjacent to flame, cultured in the plates containing $20 \mathrm{cc}$ Mueller-Hinton agar, and incubated at $37^{\circ} \mathrm{C}$ overnight. The bacterial growth was measured by the number of developed colonies and reported in $\mathrm{CFU} / \mathrm{mL}$ (19).

Then, the five bacteria (E. coli, S. epidermidis, S. aureus, $Y$. enterocolitica, and $P$. aeruginosa) were inoculated on the plates containing Mueller-Hinton base medium using sterile swab and incubated at $37^{\circ} \mathrm{C}$ overnight. The bacterial growth was reported in $\mathrm{CFU} / \mathrm{mL}$.

In the plates containing Mueller-Hinton base medium and iron, the effective antibiotic discs (Gen Fanavaran Tehran, Iran) at doses of interest were placed (22). In such a way, $30 \mu \mathrm{g}$ vancomycin disc (Biomerieux, Sweden) could be used for $S$. aureus, $30 \mu \mathrm{g}$ cefazoline disc for $S$.

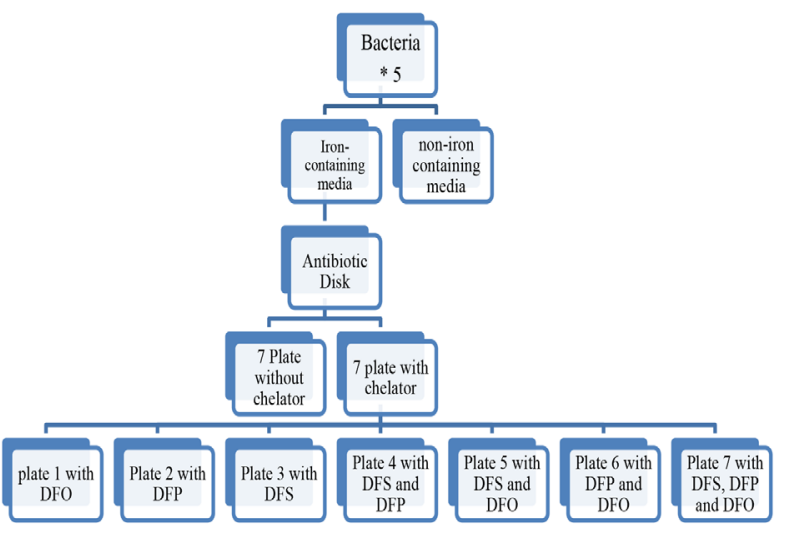

Figure 2. Antibiotic Disc Diffusion (DFO, DFS and DFP). Abbreviations: DFO, deferoxamine; DFS, deferasirox; DFP, deferiprone. 
epidermidis, $10 \mu \mathrm{g}$ gentamicin disc for $Y$. enterocolitica, 10 $\mu \mathrm{g}$ gentamicin disc for $E$. coli, and $30 \mu \mathrm{g}$ amikacin as well as $30 \mu \mathrm{g}$ ceftazidime discs separately for $P$. aeruginosa. The zone of growth inhibition (in $\mathrm{mm}$ ) was recorded after the incubation of the relevant plate at $37^{\circ} \mathrm{C}$ overnight (19).

Afterwards, the bacteria and the Mueller-Hinton base medium alongside the iron were added to the culture media and the chelators, both as single and combined, which were also added to antibiotic discs incubated at $37^{\circ} \mathrm{C}$ overnight. The zone of growth inhibition was reported in $\mathrm{mm}$ and classified as susceptible, intermediate, and resistant with reference to the Clinical and Laboratory Standards Institute (CLSI) (22) (Table 1).

\section{Results}

The growth of all bacteria increased in Mueller-Hinton agar in the presence of iron. The growth of E. coli, S. aureus, S. epidermidis, Y. enterocolitica, and P. aeruginosa increased in the plate containing Mueller-Hinton agar and antibiotic disc with addition of iron (Table 2). Overall, the growth of all bacteria was inhibited in the presence of the chelators. The growth inhibition was found different only for E. coli. In the presence of various chelators (both alone and combined), the growth of E. coli (in CFU) was inhibited differently. The lowest positive effect of the chelators on E. coli growth inhibition was derived for DFS+DFP as compared with others (Table 2). No difference was seen in the number of grown colonies of E. coli between the plates with chelators and the ones without them. In addition, increase in the zone of growth inhibition of E. coli was seen in the presence of the antibiotics and chelators, that is, they had a synergistic effect on the growth inhibition of E. coli (Table 2).

According to the guidelines of CLSI, E. coli was susceptible to gentamicin with 14-mmzone of growth inhibition. In the presence of various chelators (both single and combined), E. coli showed high susceptibility to gentamicin. The growth of S. aureus was inhibited in the presence of different chelators, with no difference in inhibition among the chelators (Table 2). No difference was

Table 1. Susceptibility of bacteria to antibiotics by zone of growth inhibition

\begin{tabular}{lccc}
\hline Bacteria and Antibiotic & Resistance & Intermediate & Susceptible \\
\hline $\begin{array}{l}\text { Escherichia coli-gentamicin } \\
\begin{array}{l}\text { Staphylococcus aureus- } \\
\text { vancomycin }\end{array}\end{array}$ & $12 \leq$ & $13-14$ & $\mathbf{1 5} \geq$ \\
$\begin{array}{l}\text { Staphylococcus epidermidis- } \\
\text { cefazoline }\end{array}$ & $14 \leq$ & $15-17$ & $\mathbf{1 8} \geq$ \\
$\begin{array}{l}\text { Yersinia enterocolitica- } \\
\text { gentamicin }\end{array}$ & $12 \leq$ & $13-14$ & $\mathbf{1 5} \geq$ \\
$\begin{array}{l}\text { Pseudomonas aeruginosa- } \\
\text { amikacin }\end{array}$ & $14 \leq$ & $15-16$ & $\mathbf{1 7} \geq$ \\
$\begin{array}{l}\text { Pseudomonas aeruginosa- } \\
\text { ceftazidime }\end{array}$ & $14 \leq$ & $15-17$ & $\mathbf{1 7} \geq$ \\
\hline
\end{tabular}

seen in the number of grown colonies of S. aureus between the plates with chelators and the ones without them. The increase in the zone of growth inhibition of S. aureus was seen in presence of antibiotics and chelators (Table 2). Based on CLSI guidelines, S. aureus was susceptible to the used antibiotic with $13-\mathrm{mm}$ zone of growth inhibition. In the presence of various chelators and antibiotics (both single and combined) in the culture medium, the zone of growth inhibition increased.

The growth of S. epidermidis was inhibited in presence of different chelators, with no significant difference in inhibition among the chelators (Table 2). No difference was seen in the number of grown colonies of S. epidermidis between the plates with chelators and the ones without them. The increase in the zone of growth inhibition of $S$. epidermidis means the inhibition of bacterial growth in presence of chelators and antibiotics (Table 2).

According to CLSI guidelines, S. epidermidis was susceptible to cefazolin with 18-mmzone of growth inhibition. In the presence of various chelators (single and combined), the zone of growth inhibition increased, and therefore S. epidermidis growth was inhibited. The growth of Y. enterocolitica was inhibited in the presence of different chelators, with the highest inhibition in the presence of DFP and DFS (Table 2). No difference was seen in the number of grown colonies of Y. enterocolitica between the plates containing chelators and the ones without them.

The growth of Y. enterocolitica was inhibited mostly in the presence of DFP+DFS+ gentamicin (the highest zone of growth inhibition). The growth of Y. enterocolitica did not change in the presence of DFS and gentamicin disc, and the zone of growth inhibition of Y. enterocolitica decreased in the presence of gentamicin and other chelators (Desferal, DFP, Desferal + DFS, Desferal +DFP, and Desferal + $\mathrm{DFP}+\mathrm{DFS})$, which represents the increased growth of $\mathrm{Y}$. enterocolitica (Table 2).

According to CLSI, Y. enterocolitica was susceptible to gentamicin with 25-mmzone of growth inhibition. In the presence of various chelators and antibiotics (both single and combined), the zone of growth inhibition increased, and therefore Y. enterocolitica growth was inhibited (Table 2). No difference was seen in the number of grown colonies of S. epidermidis between the plates with chelators and the ones without them. The growth of P. aeruginosa was inhibited in the presence of chelators (except for DFP) and amikacin, which was illustrated as increase in zone of growth inhibition in Table 2. The zone of growth inhibition or inhibition of $\mathrm{P}$. aeruginosa growth was highest in the presence of various combinations of chelators. Based on CLSI, P. aeruginosa was susceptible to the used antibiotic with 30-mmzone of growth inhibition. In the presence of various chelators (both single and combined), the zone of growth inhibition increased, and therefore P. aeruginosa growth was inhibited in the plate with the antibiotic, chelator, and iron. 
Table 2. Comparison of number of colony-forming units and zone of growth inhibition for different bacteria in medium with and without iron

\begin{tabular}{|c|c|c|c|c|}
\hline & \multicolumn{2}{|r|}{ Without iron } & \multicolumn{2}{|c|}{ With iron } \\
\hline & $\begin{array}{l}\text { Bacterial growth (CFU/ } \\
\text { mL) }\end{array}$ & $\begin{array}{l}\text { Zone of growth inhibition with antibiotic } \\
\qquad(\mathrm{mm})\end{array}$ & $\begin{array}{l}\text { Bacterial growth } \\
\quad(\mathrm{CFU} / \mathrm{mL})\end{array}$ & $\begin{array}{l}\text { Zone of growth inhibition } \\
\text { with antibiotic }(\mathrm{mm})\end{array}$ \\
\hline Escherichia coli & $30 \times 10^{3}$ & 20 & $45 \times 10^{3}$ & 14 \\
\hline Staphylococcus aureus & $24 \times 10^{3}$ & 15 & $32 \times 10^{3}$ & 13 \\
\hline Staphylococcus epidermidis & $2.3 \times 10^{4}$ & $30^{\mathrm{a}}$ & $3 \times 10^{4}$ & $25^{\text {a }}$ \\
\hline Yersinia enterocolitica & $9 \times 10^{3}$ & 28 & $14.5 \times 10^{3}$ & 25 \\
\hline $\begin{array}{l}\text { Pseudomonas aeruginosa } \\
\text { (with amikacin) }\end{array}$ & $18 \times 10^{3}$ & $31^{\mathrm{a}}$ & $23 \times 10^{3}$ & $30^{a}$ \\
\hline $\begin{array}{l}\text { Pseudomonas aeruginosa } \\
\text { (with ceftazidime) }\end{array}$ & $18 \times 10^{3}$ & $32^{\mathrm{a}}$ & $23 \times 10^{3}$ & $28^{a}$ \\
\hline
\end{tabular}

${ }^{\text {a }}$ susceptible (according to the Clinical and Laboratory Standards Institute).

Pseudomonas aeruginosa zone of growth inhibition increased in the presence of ceftazidime and different chelators, with the highest increase in the presence of Desferal and the lowest zone of growth inhibition or the highest bacterial growth in the presence of Desferal + DFS (Table 2). Based on CLSI, P. aeruginosa was susceptible to ceftazidime with 28-mmzone of growth inhibition. In the presence of various chelators plus ceftazidime, the zone of growth inhibition increased, and therefore bacterial growth was inhibited.

\section{Discussion}

In the present study, the growth of all bacteria in the culture medium and also antibiotic disc increased as iron was introduced. Clearly, studies have demonstrated that many pathogenic bacteria need external sources of iron for growth and survival (23-30). Iron as a cofactor contributes mainly to many of the reactions of oxygen transmission, metabolism, and production of energy and nucleic acid. Therefore, the growth of bacteria is likely to increase as iron is added. This study indicated that the growth of all bacteria was inhibited in the presence of different chelators, but the inhibition was different only for E. coli.

Thompson et al conducted a study on the antibacterial effect of iron chelators on nosocomial pathogens and demonstrated that DFP in tissue culture medium inhibited the growth of E. coli and other bacteria under study, while DFO did not do so (26). Because DFO is likely to provide iron to the bacteria by the receptor of their cognate siderophore, these findings are predictable. On the other hand, other mutant species of bacteria need $0.05 \mathrm{~mm}$ iron for growth (31), and therefore resistant species should be further studied.

For $S$. aureus, the growth was inhibited in the presence of various chelators in the present study. In a study on the effect of DFP on in vitro growth of staphylococci, the growth of S. aureus was inhibited in the presence of
DFP, which could be considered for the patients with iron overload (32). The results of the study conducted by Thompson et al were consistent with the ones of the present study. Moreover, DFO had no inhibitory effect on $S$. aureus growth because it facilitates the transmission of iron for bacterial growth (26). S. aureus is actively involved in the absorption of iron in iron-limited environments by producing siderophore (33), which probably leads to inconsistency of the response to DFO. The findings of another study indicated that the reason for the increased growth of $S$. aureus in the presence of Desferal is the use of DFO by the bacteria in ATP-binding cassette transport system for further use of environmental iron (34).

For S. epidermidis, the growth was inhibited in the presence of various types of chelators, with no remarkable difference among them. In a study on the effects of DFP and DFO on the in vitro growth of various staphylococci, Kim and Shin demonstrated that S. epidermidis was not able to absorb iron and growth in the medium containing the transferrin-binding iron (non-free iron). Moreover, the growth of $S$. epidermidis was inhibited in the presence of Desferal. S. epidermidis also responded to DFP in the culture medium which acted as an inhibitory agent for its growth (32). In another study, the growth of S. epidermidis was inhibited in the presence of the chelator and the inhibitory effect was removed when iron was introduced (35). Anyway, S. epidermidis exhibits a better growth in the patients with increased blood iron (36), and therefore this finding could not be considered surprising. On the other hand, S. epidermidis could be susceptible to the chelator and gene variations probably have not yet been developed to give this property to $S$. epidermidis.

For Y. enterocolitica, the growth was inhibited when different types of chelators were added to Mueller Hinton agar and iron. In addition, Y. enterocolitica showed different responses to growth inhibition in different media.

The zone of growth inhibition of Y. enterocolitica 
decreased in the presence of gentamicin and other chelators (Desferal, DFP, Desferal + DFS, Desferal + DFP, and Desferal + DFP + DFS). This means that the growth of Y. enterocolitica increased, which could be explained by the factors associated with doses of chelators, the type of antibiotic, or virulent properties of the used strain. DFP inhibited the growth of Y. enterocolitica by decreasing iron availability, which is consistent with other studies (35). The study conducted by Chan et al demonstrated that DFO could increase the risk of infection with Y. enterocolitica, while DFP did not contribute positively to both in vivo and in vitro growth of $Y$. enterocolitica (17). These findings on Y. enterocolitica in different media could be explained by the variety of doses of chelators and siderophoreproducing property of Y. enterocolitica (37). Yersiniabactin is a siderophore which has been initially detached from Y. enterocolitica. In addition to having the ability to absorb iron, this siderophore can bind to copper to prevent bacterial death (38).

In the present study, the growth of $\mathrm{P}$. aeruginosa was inhibited in the presence of chelators (except for DFP) and amikacin. The study conducted by van Asbeck et al also indicated that DFO exerted inhibitory effect on $\mathrm{P}$. aeruginosa growth in the absence of iron (35). Moreover, the study conducted by Thompson et al indicated that DFP had an inhibitory effect on the growth of P. aeruginosa, but DFO did not (26). The siderophore in P. aeruginosa, called pyoverdine, could help to explain the difference in response to chelators (39).

In the present study, the growth of E. coli, S. epidermidis, S. aureus, and P. aeruginosa was inhibited in the presence of used antibiotic discs and different chelators. The growth of Y. enterocolitica was inhibited in the presence of gentamicin and combination of DFP and DFS. van Asbeck et al and Hartzen et al also found that DFO and antibiotics could have a synergistic effect on growth inhibition of bacteria $(35,40)$. Notably, the in vivo combination of chelators and antibiotics should be used cautiously, which deserves detailed in-depth studies.

Overall, iron chelators prevent bacterial growth by preventing iron absorption, but the difference in the effect on different bacteria among the chelators could be due to the difference in the interaction of the type of siderophore in the cell wall, the type of chelating drug, and the mutant strains of the bacteria.

\section{Conclusion}

Due to the affinity of iron with various bacteria and the positive effect of chelators through the decrease in iron availability and the synergistic effect with antibiotics, it seems logical to recommend that chelators should be used for thalassemia patients with bacterial septicemia. However, as the growth of Y. enterocolitica increased in the presence of Desferal, DFP should be prescribed instead of Desferal. Future studies are recommended to investigate various bacteria and antibiotic-resistant strains as well as chelators at various doses with regard to bacterial growth.

Conflict of Interests

The authors have no conflict of interests.

Ethical considerations

This project was approved by the Ethics Committee of Research and Technology Department, affiliated with Shahrekord University of Medical Sciences (IR.SKUMS.REC.1393.9.13).

\section{Acknowledgements}

The authors of the present study would like to thank the support received from Dr. Farhad Safarpoor Dehkordi in the University of Tehran, Iran.

We also would like to thank Research and Technology Department of Shahrekord University of Medical Sciences for providing a grant for this study (1784).

References

1. Galanello R, Origa R. Beta-thalassemia. Orphanet J Rare Dis. 2010;5:11. doi: 10.1186/1750-1172-5-11.

2. World Health Organization (WHO). Genomic resource centre. Geneva: WHO; 2015. [cited 201513 August]. Available from: http://www.who.int/genomics/public/geneticdiseases/en/ index2.html.

3. Cappellini MD. Exjade(R) (deferasirox, ICL670) in the treatment of chronic iron overload associated with blood transfusion. Ther Clin Risk Manag. 2007;3(2):291-9. doi: 10.2147/ tcrm.2007.3.2.291.

4. Mariani R, Trombini P, Pozzi M, Piperno A. Iron metabolism in thalassemia and sickle cell disease. Mediterr J Hematol Infect Dis. 2009;1(1):e2009006. doi: 10.4084/mjhid.2009.006.

5. Melchiori L, Gardenghi S, Rivella S. Beta-thalassemia: HiJAKing ineffective erythropoiesis and iron overload. Adv Hematol. 2010;2010:938640. doi: 10.1155/2010/938640.

6. Inati A, Noureldine MA, Mansour A, Abbas HA. Endocrine and bone complications in beta-thalassemia intermedia: current understanding and treatment. Biomed Res Int. 2015;2015:813098. doi: 10.1155/2015/813098.

7. Paksu MS, Karli A, Paksu S, Guney AK, Ozsevik SN, Belet N. Fatal sepsis in a child with thalassemia major due to Serratia marcescens. Pediatr Int. 2014;56(5):796-7. doi: 10.1111/ ped.12492.

8. Sari TT, Gatot D, Akib AA, Bardosono S, Hadinegoro SR, Harahap $A R$, et al. Immune response of thalassemia major patients in Indonesia with and without splenectomy. Acta Med Indones. 2014;46(3):217-25.

9. Atefi A, Binesh F, Hashemi A, Atefi A, Aminorroaya M. Seroprovalence of herpes simplex1, 2 lgG antibodies in patients with beta thalassemia in a major tertiary care hospital located in Yazd, Iran. Iran J Ped Hematol Oncol. 2014;4(2):64-7.

10. Auxiliadora-Martins M, Bellissimo-Rodrigues F, Viana JM, Teixeira GC, Nicolini EA, Cordeiro KS, et al. Septic shock caused by Plesiomonas shigelloides in a patient with sickle beta-zero thalassemia. Heart Lung. 2010;39(4):335-9. doi: 10.1016/j.hrtlng.2009.06.015.

11. Haddad A, Tyan P, Radwan A, Mallat N, Taher A. betaThalassemia Intermedia: A Bird's-Eye View. Turk J Haematol. 2014;31(1):5-16. doi: 10.4274/Tjh.2014.0032.

12. Boonchooduang N, Louthrenoo O, Choeyprasert W, Charoenkwan P. Health-Related Quality of Life in Adolescents with Thalassemia. Pediatr Hematol Oncol. 2015;32(5):341-8. doi: 10.3109/08880018.2015.1033795. 
13. Mishra AK, Tiwari A. Iron overload in Beta thalassaemia major and intermedia patients. Maedica (Buchar). 2013;8(4):328-32.

14. Bazrgar M, Peiravian F, Abedpour F, Karimi M. Causes for hospitalization and death in Iranian patients with betathalassemia major. Pediatr Hematol Oncol. 2011;28(2):134-9. doi: 10.3109/08880018.2010.503336.

15. Islam S, Jarosch S, Zhou J, Parquet Mdel C, Toguri JT, Colp $\mathrm{P}$, et al. Anti-inflammatory and anti-bacterial effects of iron chelation in experimental sepsis. J Surg Res. 2016;200(1):26673. doi: 10.1016/j.jss.2015.07.001.

16. Sakran W, Levin C, Kenes Y, Colodner R, Koren A. Clinical spectrum of serious bacterial infections among splenectomized patients with hemoglobinopathies in Israel: a 37-year followup study. Infection. 2012;40(1):35-9. doi: 10.1007/s15010011-0178-5.

17. Chan GC, Chan S, Ho PL, Ha SY. Effects of chelators (deferoxamine, deferiprone and deferasirox) on the growth of Klebsiella pneumoniae and Aeromonas hydrophila isolated from transfusion-dependent thalassemia patients. Hemoglobin. 2009;33(5):352-60. doi: 10.3109/03630260903211888.

18. Sumera A, Radhakrishnan A, Baba AA, George E. Review: Betathalassemia and molecular chaperones. Blood Cells Mol Dis 2015;54(4):348-52. doi: 10.1016/j.bcmd.2015.01.008.

19. Mahon CR, Lehman DC, Manuselis G. Textbook of Diagnostic Microbiology. 4th ed. London: Elsevier Health Sciences; 2014.

20. Ferrer P, Tripathi AK, Clark MA, Hand CC, Rienhoff HY, Jr., Sullivan DJ Jr. Antimalarial iron chelator, FBS0701, shows asexual and gametocyte Plasmodium falciparum activity and single oral dose cure in a murine malaria model. PLoS One. 2012;7(5):e37171. doi: 10.1371/journal.pone.0037171.

21. Jabado N, Cuellar-Mata P, Grinstein S, Gros P. Iron chelators modulate the fusogenic properties of Salmonella-containing phagosomes. Proc Natl Acad Sci U S A. 2003;100(10):612732. doi: 10.1073/pnas.0937287100.

22. Clinical and Laboratory Standards Institute (CLSI). Performance standards for antimicrobial disk susceptibility tests; approved standard. 9th ed. Wayne, PA: CLSI; 2006.

23. Crusio R, Rao S, Changawala N, Paul V, Tiu C, van Ginkel J, et al. Epidemiology and outcome of infections with carbapenemresistant gram-negative bacteria treated with polymyxin B-based combination therapy. Scand J Infect Dis. 2014;46(1):18. doi: 10.3109/00365548.2013.844350.

24. Sutak R, Lesuisse E, Tachezy J, Richardson DR. Crusade for iron: iron uptake in unicellular eukaryotes and its significance for virulence. Trends Microbiol. 2008;16(6):261-8. doi: 10.1016/j. tim.2008.03.005

25. Skaar EP. The battle for iron between bacterial pathogens and their vertebrate hosts. PLoS Pathog. 2010;6(8):e1000949. doi: 10.1371/journal.ppat.1000949.

26. Thompson MG, Corey BW, Si Y, Craft DW, Zurawski DV. Antibacterial activities of iron chelators against common nosocomial pathogens. Antimicrob Agents Chemother.
2012;56(10):5419-21. doi: 10.1128/aac.01197-12.

27. Lin L, Pantapalangkoor P, Tan B, Bruhn KW, Ho T, Nielsen T, et al. Transferrin iron starvation therapy for lethal bacterial and fungal infections. J Infect Dis. 2014;210(2):254-64. doi: 10.1093/infdis/jiu049.

28. Ishida $\mathrm{JH}$, Johansen KL. Iron and infection in hemodialysis patients. Semin Dial. 2014;27(1):26-36. doi: 10.1111/ sdi.12168.

29. Silva-Gomes S, Vale-Costa S, Appelberg R, Gomes MS. Iron in intracellular infection: to provide or to deprive? Front Cell Infect Microbiol. 2013;3:96. doi: 10.3389/fcimb.2013.00096.

30. Parrow NL, Fleming RE, Minnick MF. Sequestration and scavenging of iron in infection. Infect Immun. 2013;81(10):3503-14. doi: 10.1128/iai.00602-13.

31. Hartmann A, Braun V. Iron uptake and iron limited growth of Escherichia coli K-12. Arch Microbiol. 1981;130(5):353-6. doi: 10.1007/bf00414599.

32. Kim CM, Shin SH. Effect of iron-chelator deferiprone on the in vitro growth of staphylococci. J Korean Med Sci. 2009;24(2):289-95. doi: 10.3346/jkms.2009.24.2.289.

33. Beasley FC, Heinrichs DE. Siderophore-mediated iron acquisition in the staphylococci. J Inorg Biochem. 2010;104(3):282-8. doi: 10.1016/j.jinorgbio.2009.09.011.

34. Sebulsky MT, Heinrichs DE. Identification and characterization of fhuD1 and fhuD2, two genes involved in ironhydroxamate uptake in Staphylococcus aureus. J Bacteriol. 2001;183(17):4994-5000. doi: 10.1128/jb.183.17.49945000.2001 .

35. van Asbeck BS, Marcelis JH, van Kats JH, Jaarsma EY, Verhoef J. Synergy between the iron chelator deferoxamine and the antimicrobial agents gentamicin, chloramphenicol, cefalothin, cefotiam and cefsulodin. Eur J Clin Microbiol. 1983;2(5):4328. doi: 10.1007/bf02013900.

36. Matinaho S, von Bonsdorff L, Rouhiainen A, Lonnroth M, Parkkinen J. Dependence of Staphylococcus epidermidis on non-transferrin-bound iron for growth. FEMS Microbiol Lett. 2001;196(2):177-82. doi: 10.1111/j.1574-6968.2001. tb10561.x.

37. Lesic B, Foulon J, Carniel E. Comparison of the effects of deferiprone versus deferoxamine on growth and virulence of Yersinia enterocolitica. Antimicrob Agents Chemother. 2002;46(6):1741-5. doi: 10.1128/aac.46.6.1741-1745.2002.

38. Perry RD, Fetherston JD. Yersiniabactin iron uptake: mechanisms and role in Yersinia pestis pathogenesis. Microbes Infect. 2011;13(10):808-17. doi: 10.1016/j.micinf.2011.04.008.

39. Rahav G, Volach V, Shapiro M, Rund D, Rachmilewitz EA, Goldfarb A. Severe infections in thalassaemic patients: prevalence and predisposing factors. $\mathrm{Br} J$ Haematol. 2006;133(6):667-74. doi: 10.1111/j.1365-2141.2006.06082.x.

40. Hartzen SH, Frimodt-Moller N, Thomsen VF. The antibacterial activity of a siderophore. 2. The influence of deferoxamine alone and combined with ascorbic acid on the activity of antibiotics against Staphylococcus aureus. Apmis. 1991;99(10):879-86. 